\title{
Delécluze e Leonardo
}

\section{Wilma Proglio}

\section{(2) OpenEdition}

\section{Journals}

\section{Edizione digitale}

URL: http://journals.openedition.org/studifrancesi/34112

DOI: 10.4000/studifrancesi.34112

ISSN: 2421-5856

\section{Editore}

Rosenberg \& Sellier

\section{Edizione cartacea}

Data di pubblicazione: 1 novembre 2005

Paginazione: 325-337

ISSN: 0039-2944

\section{Notizia bibliografica digitale}

Wilma Proglio, «Delécluze e Leonardo», Studi Francesi [Online], 146 (XLIX | II) | 2005, online dal 30

novembre 2015, consultato il 18 avril 2021. URL: http://journals.openedition.org/studifrancesi/34112 ; DOI: https://doi.org/10.4000/studifrancesi.34112

\section{(c) $(i)(9)$}

Studi Francesi è distribuita con Licenza Creative Commons Attribuzione - Non commerciale - Non opere derivate 4.0 Internazionale. 


\section{Delécluze e Leonardo}

Nel panorama della letteratura artistica della prima metà dell'Ottocento, riveste particolare interesse la personalità di Étienne-Jean Delécluze. Dopo aver svolto in prima persona e con notevole successo l'attività di pittore, egli si dedicò esclusivamente alla letteratura e alla critica d'arte, militando tra le fila dei sostenitori del classicismo ed opponendosi alle novità di quella che egli definiva la «bourrasque romantique». Nonostante la divergenza di vedute, tuttavia, Delécluze fu amico di esponenti di primo piano della nuova letteratura, tra cui Mérimée e Stendhal. Sainte-Beuve commentò tale contraddizione affermando che il nostro autore era «assez bienveillant pour les personnes, sans quartier pour les principes». Il grande critico era, inoltre, un estimatore di Delécluze, di cui apprezzò particolarmente il romanzo Mademoiselle Justine de Liron, che venne pubblicato insieme agli altri suoi romanzi La première Communion e Le lis de l'eau de Yng-Li nella raccolta Romans, contes et nouvelles (1843). Delécluze fece, inoltre, una serie di studi con l'intento di formare un quadro storico del Medioevo rimasto però incompleto; i personaggi da lui presi in esame per questo lavoro furono Gregorio VII, S. Francesco d'Assisi, S. Tommaso, Orlando e Dante, del quale tradusse la Vita Nuova. Due tra le sue opere più importanti sono Louis David, son école et son temps ed il Souvenir de soixante années ${ }^{1}$. Delécluze scrisse anche numerosi articoli su alcuni grandi maestri della storia dell'arte, tra cui il saggio intitolato Léonard de Vinci, pubblicato sulla rivista L'Artiste del 1841, il quale risulta particolarmente interessante, per le novità che lo differenziano dagli studi cronologicamente precedenti su Leonardo.

Occorre premettere, innanzi tutto, che in questo saggio Delécluze ha voluto tratteggiare un profilo biografico ed umano di Leonardo, piuttosto che emettere una serie di giudizi sulla sua arte. L'obiettivo finale dell'autore, inoltre, è quello di dimostrare, attraverso la parabola artistica e umana del maestro toscano, il sodalizio tra l'arte e la scienza.

L'articolo comincia con un poetico paragone tra l'arte e i fiori: «de temps immémorial on admire les fleurs, en se demandant à quelle fin le Créateur les a faites si belles, mystère impénétrable [...]. Le rang que tiennent les fleurs dans le développement des plantes est occupé, dans la série des inventions humaines, par les Beaux-Arts, cet accident plein d'éclat qui se manifeste comme l'une des formes successives que prend la pensée pour se dérouler et tendre à la perfection» ${ }^{2}$. L'arte, quindi, secondo l'autore,

(1) Étienne-Jean Delécluze (1781-1863), figlio di un architetto, fu uno dei primi allievi di David. Nei Salons allestiti tra il 1808 ed il 1814 espose quadri di soggetto per lo più mitologico, come il rapimento di Europa ed il rapimento di Elena. Collaborò a diversi periodici, in particolare al Journal des débats, per il quale ebbe l'incarico di scrivere i resoconti dei Salons tra il 1824 ed il 1855 . Sempre per questo giornale egli seguì le rappresentazioni del Théâtre Italien. Possessore di un'ingente fortuna, soggiornò a lungo in Inghilterra ed in Italia. (La Grande Encyclopèdie, sous la direction de M. Berthelot, Paris, Lamirault et Compagnie, 1885/ 1892, vol. III, p. 1176-1177).

Per avere un quadro del clima storico-artistico e critico in cui si formò, visse ed operò Delécluze, si rimanda ai seguenti testi: G. BAzIN, Histoire de l'bistoire de l'art, Paris, Albin Michel, 1986, p. 114-141; F. HASKELL, Un monument et ses mystères. L'art français et l'opinion anglaise dans la première moitié du XIX siècle, sta in «Revue de l'Art», no 30, Paris, CNR, 1975, p. 61-76 ; A. PINELLI, Nel segno di Giano. Passato e futuro nell'arte europea tra Sette e Ottocento, Roma, Carocci, 2000, pp. 289-370 (da segnalare è l'ampio spazio che Pinelli dedica all'opera David, son école et son temps di Delécluze, considerata un testo fondamentale per conoscere il grande pittore francese, grazie al suo valore di testimonianza diretta, L. Venturi, Storia della critica d'arte, Torino, Einaudi, 1964, p. 172-197).

(2) É.-J. Delecluze, Léonard de Vinci (14521519), Paris, Imprimerie de Schneider et Langrand, Extrait du journal «L'Artiste», 1841, p. 5-6. Tutte le citazioni riportate in questo articolo rispettano la grafia originale dei testi da cui sono attinte. 
non è un fine, ma un mezzo per sviluppare appieno le potenzialità umane, così come il fiore è solo uno stadio intermedio che ha come meta finale la pianta. Quale sia, in questa metafora, l'equivalente della pianta, Delécluze lo svelerà alla fine del suo saggio, dopo aver analizzato la figura e l'opera di Leonardo.

L'autore prosegue affermando che le arti non hanno conosciuto lo stesso sviluppo presso tutti i popoli, ma che le epoche in cui esse conobbero il maggior splendore furono la Grecia del tempo di Pericle e il periodo che va dal XIII secolo fino al 1561. Questi due momenti della storia dell'umanità sarebbero, secondo Delécluze, l'eco l'uno dell'altro e l'energia che in quelle fasi storiche era animato gli artisti, «est une secousse indispensable et salutaire donnée à l'espèce humaine, comme les battements réglés du cœur qui entretiennent la vie dans le corps des animaux»³.

L'autore, postosi l'obiettivo di illustrare il ruolo delle arti figurative nel movimento intellettuale del Rinascimento, riconosce di aver avuto difficoltà nello scegliere la personalità che più di ogni altra potesse facilitargli il compito. Alla fine ha optato per Leonardo. Questo artista viene da lui considerato, in altri termini, più di ogni altro rappresentativo del suo tempo ${ }^{4}$.

Circa le origini del pittore toscano, Delécluze afferma che egli nacque a Vinci nel 1452 e che «il était fils naturel d'un notaire, dont les descendants vivaient encore dans une honnête médiocrité» ${ }^{5}$; il biografo prosegue elencando i doni di cui la natura fu prodiga nei suoi confronti: la bellezza fisica, il buon carattere e la predisposizione per tutti i campi del sapere. Nella bottega del Verrocchio, Leonardo non seguì l'itinerario di studi degli altri allievi, poiché il suo maestro, «dont les talents étaient trop faibles pour diriger un tel élève, avait cependant assez de tact pour reconnaître l'excellence de son mérite, et, en homme de sens, il laissa le jeune Léonard étudier comme il l'entendait» ${ }^{6}$. L'autore francese elogia la grande umiltà di Verrocchio, che avendo riconosciuto l'eccellenza di Leonardo dopo il suo contributo al Battesimo di Cristo, abbandonò la pittura. L'interpretazione di questo gesto in chiave positiva costituisce una novità, dal momento che alcuni precedenti biografi di Leonardo lo avevano, invece, considerato una reazione suscitata dall'invidia e dal risentimento del Verrocchio nei confronti del giovane allievo ${ }^{7}$.

(3) Ibidem, p. 7-8.

(4) Prima di entrare nel vivo dell'oggetto del suo saggio, Delécluze traccia una breve storia dell'arte cristiana, affermando che in origine essa adottò alcune forme e alcuni elementi di quella pagana. In seguito si discusse la questione dell'iconoclastia, che venne affrontata nel secondo concilio di Nicea del 787, nel quale venne ammesso l'uso delle immagini finalizzate all'incremento della devozione e all'apprendimento delle sacre scritture. Da quel momento cominciò lo sviluppo dell'arte di soggetto religioso, che rimase l'unica per molto tempo. In Italia fu con Cimabue, Nicola Pisano e poi soprattutto con Giotto, che gli artisti cominciarono a coltivare nuovi aspetti, quali l'imitazione del naturale e l'espressione delle passioni dell'animo. Dante contribuì al superamento degli schemi artistici devozionali finora adottati, perché con il suo poema inventò una nuova mitologia cristiana. Brunelleschi, Donatello e Masaccio diedero poi un grande impulso allo sviluppo delle arti figurative, anche grazie alla scoperta di alcuni capolavori della statuaria greca, che cominciarono a fungere da modello. Quando nacque Leonardo i grandi intelletti d'Italia erano quindi impegnati a far rivivere la grandezza dell'arte greca e a ricercare la verità attraverso la riscoperta della filosofia platonica. Ibidem, p. 9-13.

(5) Ibidem, p. 13.

(6) Ibidem, p. 14.

(7) Il primo a commentare la decisione di Verrocchio come una conseguenza della sua invidia fu Giorgio Vasari, il quale scrisse: «[Leonardo] acconciossi dunque [...] a l'arte con Andrea del Verrocchio, il quale, faccendo una tavola dove San Giovanni battezzava Cristo, Lionardo lavorò un angelo, che teneva alcune vesti; e benchè fosse giovanetto, lo condusse di tal maniera, che molto meglio de le figure d'Andrea stava l'angelo di Lionardo. Il che fu cagione ch'Andrea mai più non volle toccar colori, sdegnatosi che un fanciullo ne sapesse più di lui» (G. VASARI, Vite dè più eccellenti pittori, scultori e architettori, testo dell'edizione giuntina, Milano, Edizioni per il Club del Libro, 1963, vol. III, p. 391). Di tale episodio parlarono in termini analoghi parecchi biografi successivi, per esempio Dézallier d'Argenville, il quale scrisse: «cet ange étoit si parfait et si supérieur aux autres figures, que son maître, piqué de se voir ainsi effacé par un jeune homme, ne voulut plus manier le pinceau» (D. D'Argenville, Abrégé de la vie des plus fameux 
Delécluze prosegue riportando l'aneddoto vasariano della rotella di legno che il padre di Leonardo consegnò al figlio, per conto di un suo lavorante, affinché la decorasse. Leonardo, dopo aver levigato il legno, vi dipinse sopra un mostro, per la cui realizzazione egli portò nel suo studio rettili e insetti dai quali prese spunto per comporre un essere terrificante, comparabile all'antica Medusa. L'autore francese afferma che «la peinture de ce monstre imaginaire, résultat combiné de l'étude de plusieurs animaux, est certainement un trait qui caractérise avec force la double faculté d'artiste et de savant que Léonard développa avec tant d'éclat par la suite» ${ }^{8}$. Secondo Delécluze, dal modo in cui Leonardo ha realizzato quest'opera traspare il suo interesse scientifico, dal momento che egli ha combinato insieme le caratteristiche di diversi esseri viventi.

L'autore ricorda poi che Leonardo dipinse veramente una testa di Medusa «qui orne la galerie de Florence, fantaisie scientifique de cet habile peintre, observateur si originel de la nature»". La vicenda della Medusa di Leonardo è un caso molto interessante della storia dell'arte: costituisce, infatti, una sorta di falso storico. Le notizie relative a questo quadro (che i cataloghi ufficiali di Leonardo annoverano tra le opere del maestro andate perdute) si arrestano al 1533, anno in cui viene registrato nell'inventario della guardaroba medicea ${ }^{10}$. Nell'Ottocento, tuttavia, alcuni studiosi credettero di poter identificare la Medusa leonardesca in un dipinto dal medesimo soggetto conservato agli Uffizi. Uno dei primi viaggiatori a darne notizia fu CharlesNicolas Cochin, il quale, nel suo celebre Voyage d'Italie, pietra miliare della letteratura di viaggi tra il Sette e l'Ottocento, dichiarò di aver visto: «une tête de Meduse coupée, de Léonard de Vinci, bien dessinée, d'un beau et grand caractère: la couleur en est toute passée» ${ }^{11}$. L'opera alla quale alludeva Cochin è in realtà un dipinto fiammingo del XVII secolo (fig. 1). L'attribuzione di questo quadro a Leonardo divenne corrente dopo il 1783, quando fu consacrata da Zacchiroli nella sua Description de la Galerie Royale de Florence. Nel corso dell'Ottocento questa ritrovata, presunta opera leonardesca esercitò una grande suggestione presso i poeti e gli scrittori romantici, che in essa individuarono un tentativo di far coesistere l'orrido ed il bello ${ }^{12}$. Il fascino macabro di quel soggetto, nel quale viene catturato il momento di passaggio dalla vita alla morte, evidente nell'ultimo respiro che esala dalla bocca della Medusa e nei

peintres, Paris, chez Debure l'ainé, 1745, pp. 72). Anche Lévesque riportò la medesima considerazione: «Verrocchio, son maître, se reconnaissant vaincu par son élève, abandona les pinceaux et se borna uniquement à la Sculpture» (P. H. WATELETP. C. Levesque, Dictionnaire des arts de peinture, sculpture et gravure, Paris, Imprimerie de Prault l'ainé, 1792, p.24). Gault de Saint-Germain, invece, mise l'accento sul dolore provato dal Verrocchio nel vedersi superato da un giovane allievo: «il [Léonard] peignit dans ce tableau, par l'invitation de son maître, la figure d'un ange, qui surpasseoit tant en beauté le reste du tableau, qu'André en eut un tel chagrin qu'il quitta la palette, et n'osa plus la reprendre» (P.-M. GAUlt DE SaInT-Germain, Traité de la Peinture de Léonard de Vinci, précédé de la vie de l'auteur et du catalogue de ses ouvrages, avec des notes et des observations, Paris, chez Perlet, 1803, p. XXXI).

(8) Ë.-J. Delécluze, op. cit., p. 15.

(9) Ibidem.

(10) P. C. Marani, Leonardo. Catalogo completo, Firenze, Cantini, 1989, p. 125.

(11) C. Michel, Le Voyage d'Italie de Charles-
Nicolas Cocbin (1758), École française de Rome, 1991, pp. 203-204.

(12) L'interesse che si concentra attorno a quest'opera può essere messo in relazione con la teorizzazione del grottesco operata da Victor Hugo nella prefazione di Cromwell del 1827. In quelle pagine, divenute il manifesto del dramma romantico, il grande scrittore definisce il grottesco come «moyen de contraste [...], la plus riche source que la nature puisse ouvrir à l'art [...] Cette beauté universelle que l'antiquité répandait solennellement sur tout n'était pas sans monotonie; la même impression, toujours répétée, peut fatiguer à la longue. Le sublime sur le sublime produit malaisément un contraste, et l'on a besoin de se reposer de tout, même du beau. Il semble, au contraire, que le grotesque soit un temps d'arrêt, un terme de comparaison, un point de départ d'où l'on s'élève vers le beau avec une perception plus fraîche et plus excitée. [...] Le beau n'a qu'un type, le laid en a mille.» (V Hugo, Préface de Cromwell, Paris, Larousse, 1949, p. 2829). In questa nuova prospettiva, anche le creature più orribili e gli aspetti più degradati della realtà diventano parti essenziali dell'arte. 


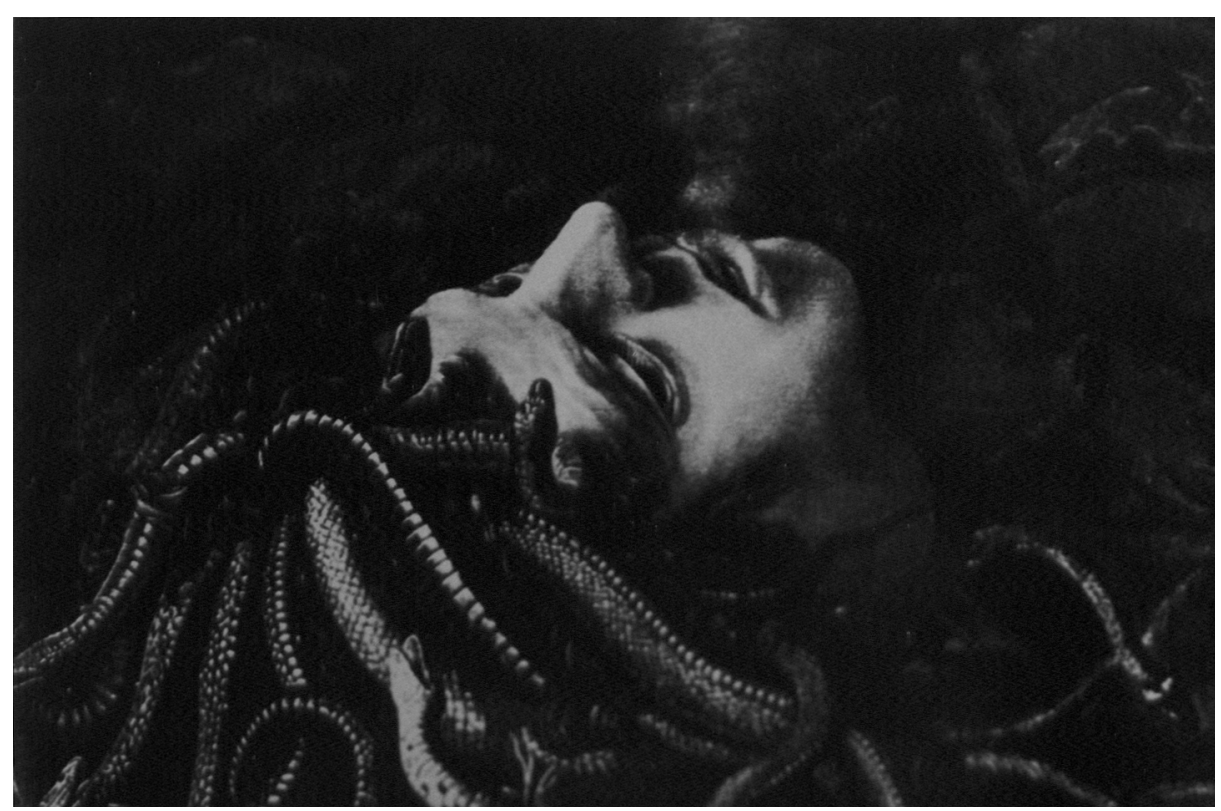

movimenti contratti delle serpi, venne considerato confacente a Leonardo, soprattutto in considerazione della sua ansia di sperimentare e stupire ${ }^{13}$. Nel 1899, tuttavia, Eugène Mûntz scoprì la verità e, suo malgrado, respinse definitivamente la seducente attribuzione di tale quadro a Leonardo ${ }^{14}$.

Un altro aspetto dell'artista messo in luce da Delécluze è, inoltre, la sua convivialità e la sua capacità di allietare le compagnie: «Léonard, malgré le nombre et l'importance de ses occupations sérieuses, était encore un des élégants de la ville de Florence, où on le recherchait avec empressement, même pour ses talents de société; car [...] il était bon musicien, improvisait des vers en chantant et en s'accompagnant sur la lyre» ${ }^{15}$. L'autore riporta anche alcuni esperimenti eseguiti da Leonardo al solo scopo di divertirsi, come quello consistente nel gonfiare d'aria le viscere di un animale macellato, fino a riempire in questo modo un'intera stanza (tale aneddoto è peraltro narrato anche da Vasari). Al tempo in cui scrive Delécluze, la riscoperta della componente ilare della personalità di Leonardo è in realtà piuttosto recente, poiché risale all'opera di Gault de Saint-Germain, Traité de la Peinture de Léonard de Vinci, del 1803. In questo testo, che consiste in una interessante traduzione del Libro di Pittura leonardesco, Gault afferma che:

(13) Ecco le parole con cui Stendhal descrisse questo dipinto: «On n'aperçoit le visage qu'en raccourci. Il semble que le peintre ait plus cherché à rendre l'horreur de la chevelure de la fille de Phorcus, que l'horreur de sa physionomie. La vie est dans les couleuvres vertes qui s'agitent sur sa tête. Pour elle, il ne l'a pas peinte morte, mais mourante: son œil terne n'est pas encore fermé; elle rend le dernier soupir, et l'on voit le souffle impur qui s'exale de sa bouche» (STENDHAL, Historie de la Peinture en Italie, Paris, Gallimard, 1996, p. 174-175).

(14) Circa la fortuna critica e le attribuzioni di questo dipinto si rimanda a R. TURNER, Words and pictures: the birth and death of Leonardo's Medusa, sta in «Arte Lombarda», $n^{\circ} 66$, volume che ospita gli atti del convegno «Umanesimo, problemi aperti», 1983, p. 103-111.

(15) É.-J. DelÉCLuZE, op. cit., pp. 16. 
«la gaité, chez le penseur, est un sentiment qui naît de la confiance dans ses facultés, et le fruit de la réflexion; elle lui sert encore à faire digression avec lui-même. Léonard invite l'homme laborieux à s'y livrer quelquefois. "Encore (dit-il) il sera fort utile de quitter son travail et de s'aller divertir un peu, parce qu'au retour on aura l'esprit plus libre: au contraire, une application trop grande et trop assidue, appesantit l'esprit et le fait tomber dans de grosses erreurs" ${ }^{16} \gg$.

È significativo osservare come i biografi settecenteschi di Leonardo, quali Lacombe, Dézallier d'Argenville, Mariette e tutti coloro che, più o meno diffusamente avevano parlato di Leonardo, tra cui Watelet e Lévesque, abbiano taciuto la componente irrazionale del carattere di Leonardo, pur se nota fin da Vasari, per privilegiare, invece, la celebrazione del suo talento e l'impegno alacremente profuso dall'artista nel progresso del sapere. Tale scelta, tuttavia, risulta del tutto consona con lo spirito dell'Illuminismo. Il nuovo secolo, invece, inizia con una maggior apertura anche verso i lati meno logici e razionali della personalità del maestro, anticipando così l'interesse per gli aspetti meno ortodossi di Leonardo, interesse che si accentrerà in età romantica. Nel caso di Delécluze, la scelta di mettere l'accento anche sulla ilarità di Leonardo risponde all'esigenza di tracciare un quadro dell'artista il più possibile completo e corretto.

Verso il 1481 Leonardo, avendo notato la fredda accoglienza tributata a due suoi progetti da parte dei Medici, decise, com'è noto, di lasciare Firenze per Milano. È interessante l'osservazione di Delécluze a proposito della condotta dei signori di Firenze: «on ignore absolument pourquoi Laurent de Médicis et Pierre, son successeur, n'eurent pas l'idée de s'attacher un artiste aussi éminent» ${ }^{17}$. Quest'affermazione dell'autore denota una riflessione da parte sua sulla cornice storico-politica in cui operava l'artista, cornice che, come si avrà modo di osservare in seguito, rappresenta un oggetto di attenzione ricorrente per Delécluze. Occorre ricordare, che la storia costituisce uno degli interessi principali dell'autore che, come già si è detto, aveva in progetto di scrivere un vasto affresco storico dedicato ai protagonisti del Medioevo. Il disinteresse dei Medici nei confronti di Leonardo dà inoltre ancora più risalto alla grande stima che, invece, Francesco I nutrì per lui.

Leonardo, pertanto, cercò di essere ammesso nella cerchia di studiosi che lavoravano presso Ludovico il Moro, inviandogli una lettera che si conserva nel Codice Atlantico; in essa egli illustrava tutte le sue competenze nel campo dell'ingegneria militare e solo alla fine faceva riferimento alle opere che egli avrebbe potuto eseguire in tempo di pace, quelle, cioè, di architettura, pittura e scultura. Delécluze riporta per intero la lettera in traduzione francese ed osserva che «après l'énoncé [...] de ce qu'il était capable de faire, rien n'est si étrange que son entrée chez le prince dont il venait chercher la protection» e prosegue raccontando che il duca amava molto sentire suonare la lira e che Leonardo arrivò a Milano con lo strumento da lui fabbricato, a forma di teschio di cavallo, sul quale suonò superando in bravura tutti gli altri musicisti che erano stati chiamati a corte. Il duca, dopo aver ascoltato l'esecuzione di Leonardo, lo accolse presso di sé e lo colmò di elogi ${ }^{18}$.

Delécluze afferma che, durante la sua permanenza a Milano, Leonardo lavorò alacremente al monumento equestre di Francesco Sforza ed i suoi scritti lo testimoniano. Inoltre il pittore eseguì i ritratti delle due amanti del Moro, cioè Cecilia Gallerani e Lucrezia Crivelli e fondò l'Accademia che porta il suo nome. Quest'ultima informazione ricorreva già in Vasari, che aveva parlato di un disegno raffigurante un nodo, all'interno del quale si leggono le parole «Leonardus Vinci Accademia». È

(16) P.-M. Gault De Saint-Germain, op. cit., p. XXXIV.
(17) É.-J. DelÉCluZE, op. cit., p. 17.

(18) Ibidem, p. 20. 
certo che con questo termine non si deve intendere un'istituzione come quelle del Sei-Settecento, con statuti e rituali precisi, ma semplicemente un circolo di artisti riuniti attorno alla fama del maestro. Delécluze afferma, inoltre, che il pittore toscano aveva l'incarico di avviare alle arti figurative e alle scienze alcuni allievi prescelti dal duca e sostiene che la grande quantità di scritti di Leonardo, di cui una parte andò a costituire il Trattato di Pittura, nasce proprio dai suoi doveri di professore e dalle sue conversazioni con gli altri letterati ed artisti.

Delécluze ritiene che il sigillo della gloria di Leonardo sia il «Cenacolo», «qui serait sans doute encore étudié aujourd'hui comme l'un des chefs-d'œuvre de l'art, si l'humidité du mur sur lequel il fut exécuté, jointe à la barbarie de quelques hommes et à l'ignorance de certains autres, n'en avait pas de très-bonne heure hâté la ruine» ${ }^{19}$. Per quanto riguarda la «barbarie di certi uomini», sicuramente Delécluze si riferisce alle devastazioni compiute durante l'occupazione napoleonica, quando il refettorio di Santa Maria delle Grazie venne utilizzato come scuderia e granaio. Circa «l'ignoranza di alcuni altri uomini», è invece probabile che egli intenda l'incuria dei Domenicani. Ciò che di questo affresco colpisce di più, secondo Delécluze, è «l'absence complète de tous les signes materiels employés jusque-là pour caractériser ce qu'il peut y avoir de surnaturel dans les personnages [...]. Le Christ n'a point d'aureole, et chaque apôtre n'a sur ses vêtements ou près de lui aucun des insignes traditionnels au moyen desquels les peintres l'avaient toujours fait distinguer materiellement jusque-là» ${ }^{20}$. Per sottolineare il carattere innovativo di questa scelta, il critico ricorda che dai primi tempi del Cristianesimo fino agli allievi di Giotto, si usava distinguere l'importanza dei personaggi variandone la statura. I meriti più alti di questa composizione sono, invece, la verità con cui le passioni dell'anima sono state dipinte sui volti degli apostoli e la vastità della gamma delle loro espressioni, che vanno dai «traits bas et repoussants de Judas, jusqu’à la douceur angélique de saint Jean et à la divinité du Christ» ${ }^{21}$.

Lo scrittore afferma che della vita privata e del carattere del maestro toscano non si sa quasi nulla. Tuttavia è possibile ricavare qualche idea sul suo modo di concepire la vita e la sua professione dalle decisioni che egli prese dopo l'ingresso di Luigi XII a Milano. Quando, nel 1499, Ludovico il Moro venne condotto in esilio in Francia, Leonardo non lo seguì. La scelta di non essere fedele al suo protettore trova una spiegazione in qualche nota dei suoi manoscritti, dalle quali sembra che «Léonard, séduit par l'idée de ne pas perdre le fruit des services qu'il avait rendus à ce pays d'adoption [...], se considéra comme devant toujours être l'artiste attaché au prince, quel qu'il fût, qui gouvernerait le duché, où il se flatta encore de faire fleurir les sciences et les arts ${ }^{22}$. Quest'osservazione sul modo di concepire l'impegno dell'artista al servizio del ducato di Milano è del tutto nuova. Infatti le succitate biografie settecentesche di Leonardo si limitavano a presentarne la vita in funzione delle opere d'arte e a segnalare gli spostamenti del maestro senza motivarli e commentarli. Da parte di Delécluze, invece, c'è una profonda volontà di approfondire il contesto storico in cui Leonardo è vissuto e che certamente ha influito sulle sue scelte e sulla sua attività. Delécluze è consapevole che la vita e la produzione di Leonardo non hanno avuto un decorso dipendente solo dalla sua genialità, ma che, al contrario, essendo il maestro toscano immerso in una realtà sociale e politica concreta, egli ha avuto un'esistenza legata agli incerti destini degli Stati dell'epoca. La biografia di Delécluze, pertanto, non si configura più come una successione di aneddoti più o meno leggendari, non è neppure il racconto quasi epico di un genio perseguitato dai contemporanei per l'eccellenza dei suoi talenti, come emergeva dalla vita di Leonardo scritta da Gault de Saint-Germain

(19) Ibidem, p. 25.

(20) Ibidem.
(21) Ibidem, p. 27.

(22) Ibidem, p. 28. 
nel, bensì si viene delineando come un racconto animato da una grande coscienza storica, oltre che da una precisa volontà critica ${ }^{23}$.

Leonardo, però, si illudeva di portare avanti i suoi studi nel ducato di Milano e del suo errore si accorse presto, dal momento che «tout l'argent superflu et même le nécessaire furent employés par les vainqueurs à donner des fêtes, des bals, des tournois; ce qui fit prendre à Léonard le parti d'aller porter ses talents ailleurs. Accompagné de son élève favori, Salaï, et de son ami, frère Pacciolo le mathématicien, il partit pour Florence, où les trois exilés s'établirent $\gg^{24}$. In quella città, dice Delécluze, Leonardo eseguì allora il ritratto di Ginevra de’ Benci, la «Gioconda» e la «Vergine, Sant'Anna e il Bambino». È il Vasari che dà queste informazioni, anche se in parte sono errate perché quello di Ginevra de' Benci è un ritratto giovanile, che si può datare attorno al 1474. In una nota a fondo pagina, Delécluze specifica che questo quadro è conosciuto in Francia con il nome di «Belle Ferronnière». Da ciò emerge la confusione che regnava nella Francia del tempo a proposito di quest'ultima opera. Gault de Saint-Germain sosteneva addirittura che il quadro di Leonardo conosciuto come «Belle Ferronnière» fosse un ritratto di Anna Bolena, l'amante e poi moglie di Enrico VIII d'Inghilterra. Stando a quanto dice Gault, Leonardo avrebbe eseguito quest'opera quando la donna venne in Francia con Maria, moglie di Luigi XII ${ }^{25}$.

A questo punto Delécluze fa una lunga digressione in cui illustra la vita di Michelangelo e Raffaello, trattando anche l'opera di altri due pittori, Beato Angelico e Perugino. Le vite di Michelangelo e Leonardo si intrecciano in occasione dell'incarico, ricevuto da entrambi, di dipingere dei momenti della storia fiorentina in Palazzo Vecchio. Delécluze afferma che Leonardo scelse di rappresentare la battaglia di Anghiari e Michelangelo quella di Cascina, mentre fu il governo della città ad assegnare loro i soggetti. I cartoni preparatori per questi affreschi vengono ricordati dall'autore francese come capolavori che attrassero a Firenze visitatori illustri, tra cui il giovane Raffaello, mossi dall'intento di contemplarli e studiarli.

Dopo aver esposto dettagliatamente le vite dei più grandi artisti del Rinascimento, Delécluze sostiene la superiorità di Leonardo, affermando che «Raphaël devint savant sans s'en douter, Michel-Ange de parti pris; mais Léonard de Vinci était né tel $\gg^{26}$. L'autore ritiene infatti che Raffaello abbia iniziato la sua attività provvisto di una scarsa preparazione culturale e che poco per volta, frequentando gli ambienti romani, abbia elevato le sue conoscenze. Il volitivo Michelangelo, invece, lavorò alacremente studiando la statuaria antica e l'anatomia. Leonardo, al contrario, era nato con doti superiori. È singolare che in un saggio dedicato a Leonardo venga assegnato uno spazio così rilevante alla vita ed alle opere di artisti suoi contemporanei. Sebbene i riferimenti ad altri pittori, infatti, non manchino anche nelle altre biografie del maestro, il caso di Delécluze si contraddistingue per l'estensione dello spazio riservato loro. Il paragone di Leonardo con Michelangelo e Raffaello (che ovviamente si risolve nella

(23) Un primo approccio alla prospettiva storica nelle biografie di Leonardo è presente nell'opera di Landon: «[...] peu de temps après arriva la défaite du duc de Milan, qui fut amené prisonnier en France en 1500, et mourut à Loches. Cette disgrâce des Sforce, et les troubles qui étaient dans la Lombardie, furent cause que l'académie qui s'était établie à Milan se dissipa peu à peu» (C-P. LANDON, Vies et ceuvres des peintres les plus célèbres de toutes les écoles, Paris, chez C.-P. Landon, 1809, p. 4).

(24) É.-J. Delécluze, op. cit., p. 29. Parimenti Stendhal aveva scritto nella sua Histoire de la Peinture en Italie: «il paraît qu'il [Léonard] eut l'espoir de voir les arts fleurir de nouveau à Milan; mais, s'étant aperçu que les Français, au milieu de leurs opérations guerrières, ne voulaient que des fêtes et des intrigues avec des jolies femmes, il partit pour Florence avec son cher Salaï et son ami le géomètre Fra Paciolo» (STEND, op. cit., p. 204). È molto evidente l'analogia tra questo brano e quello di Delécluze riportato sopra e tale somiglianza può trovare spiegazione nella lettura, da parte di quest'ultimo, dell'opera di Stendhal (la cui pubblicazione risale al 1817), e nel sentimento di amicizia e stima che legava i due scrittori.

(25) P.-M. Gault Saint-Germain, op. cit., pp. LXIV-LXV.

(26) É.-J. DelÉcluze, op. cit., p. 59. 
superiorità del primo) è tipico della critica leonardesca, ma nell'opera di Delécluze il suo scopo non è solo quello di far brillare ulteriormente l'opera del maestro, bensì è ancora una volta quello di contribuire a delucidare il contesto in cui l'artista operava. L'ampio spazio assegnato alle figure di Beato Angelico e Perugino, invece, può essere dovuto alla grande rivalutazione di questi due pittori compiuta pochi anni prima da Alexis François Rio, autore dell'opera De l'Art Chrétien, la cui pubblicazione iniziò nel $1836^{27}$.

Più avanti Delécluze fa un'altra osservazione storica interessante: «il est remarquable qu'un homme aussi distingué que Léonard, né dans le sein d'une république, non-seulement n'ait, par aucun passage de ses nombreux écrits, laissé de traces d'amour ou de haine pour tel ou tel des souverains qui l'ont protégé, mais semble même absolument étranger à l'amour de la patrie» ${ }^{28}$. L'unico amore di Leonardo era quindi il progresso del sapere umano, sia in campo artistico sia in quello scientifico, indipendentemente dal potere politico sotto il quale egli conducesse i suoi studi. Nelle parole di Delécluze, tuttavia, si legge un certo disappunto nei confronti del maestro, il quale nella sua vita rimase insensibile ed indifferente alle vicende politiche del suo tempo.

In seguito Delécluze ricorda il ritorno di Leonardo a Firenze per dividere l'eredità dello zio e poi la sua breve permanenza a Roma alla corte di Leone X, dove «se forma une petite cabale favorable à Michel-Ange et à Raphä̈l» ${ }^{29}$. L'autore francese riporta anche l'episodio vasariano del quadro commissionato dal Papa, per il quale Leonardo cominciò a preparare le vernici prima ancora di averlo iniziato, suscitando così la disapprovazione del Pontefice ${ }^{30}$.

A proposito della fallimentare esperienza leonardesca a Roma, Delécluze fa una considerazione interessante e nuova. Anziché mettere in evidenza, come aveva fatto Gault de Saint-Germain, le ingiustizie abbattutesi sul maestro toscano e presentarle come la sorte di tutti gli uomini di genio, il nostro critico sofferma la sua attenzione sui monumenti romani e su Leone X, affermando che la loro gloria sarebbe stata più grande se avessero potuto fregiarsi di opere leonardesche: «Michel-Ange et Raphaël étaient bien dignes de concourir aux embellissement de Saint-Pierre de Rome et du Vatican; mais leur gloire, ainsi que celle de Léon X, serait plus grande à mon sens, si entre les peintures des Stanze et la voûte de la Sixtine, on voyait encore quelques belles compositions de Léonard de Vinci. Ce grand nom fait faute à Rome» ${ }^{31}$.

L'autore francese racconta poi l'episodio del leone meccanico, che avrebbe particolarmente colpito Francesco I, tanto da indurlo a invitare Leonardo a seguirlo: «ce fut dans une de ces occasions, qu'à Pavie, Léonard de Vinci, voulant faire sa cour au jeune monarque son protecteur, combina un lion mécanique, qui, dans la salle du banquet, marcha jusqu'auprès du roi, puis, s'étant arrêté tout à coup, se dressa, ouvrit ses flancs et laissa voir l'intérieur de son corps rempli de fleurs de lis» ${ }^{32}$. Ben presto il sovrano condusse con sé l'artista a Bologna in occasione di un incontro con Leone X. Ecco come Delécluze commenta questo episodio: «âgé de soixante-quatre ans alors, certain désormais de finir tranquillement ses jours sous la protection du roi de France, et n'ayant pas oublié le froid accueil que lui avait fait le pape, Léonard de Vinci éprouva sans doute une joie secrète en se voyant près du vainqueur, à qui

(27) Si rimanda a questo proposito alle opere di G. Bazin E L. Venturi, citate nella nota 1.

(28) É.-J. DelÉCluZE, op. cit., p. 60.

(29) Ibidem, p. 62.

(30) «Dicesi, che essendogli allogato una opera dal papa, subito cominciò a stillare olii et erbe per far la vernice; perché fu detto dal papa Leone: "Oimé costui non è per far nulla, da che comincia a pensare alla fine innanzi il principio dell'opera”» (G. VASARI, op. cit., p. 406).

(31) É.-J. DeléCLUZe, op. cit., p. 63.

(32) Ibidem, p. 64. 
Léon X, effrayé de ses succès, était obligé de faire des concessions» ${ }^{33}$. Nessuno dei precedenti biografi di Leonardo aveva riportato questo incontro, che agli occhi di Delécluze, attenti come sempre agli eventi storici che segnarono la vita dell'artista toscano, appare invece come una piccola vendetta nei confronti del Pontefice.

Leonardo partì, quindi, alla volta della Francia. Delécluze dichiara di non possedere informazioni circa le occupazioni dell'artista durante i suoi tre anni di permanenza nel castello di Cloux, nei pressi di Amboise. Il nostro autore riporta poi l'affermazione di Vasari, secondo il quale, avvicinandosi gli ultimi momenti della sua vita, Leonardo, che fino ad allora visse senza religione, cominciò ad interessarsi alla fede cattolica. Delécluze cerca di difendere Leonardo da un'immagine di empietà, tracciando un ritratto del maestro equilibrato ed obiettivo: «Léonard a donc eu dans ses mœurs des défauts condamnables; mais tout semble indiquer d'ailleurs qu'il n'y a eu rien d'excessif dans le développement de ses passions, et que sa conduite ostensible a toujours été régulière. Quant à ses écrits, on n'y trouve rien qui ait trait à la religion; on voit qui n'était sans doute pas chaud croyant, mais jamais il ne fait parade d'incredulité, même dans les nombreuses notes où il se parlait à lui-même et qu'il n'écrivait que pour lui» ${ }^{34}$. Quest'opinione relativa alla riservatezza di Leonardo in materia religiosa è condivisa anche degli studiosi più recenti; Giuseppina Fumagalli, infatti, afferma che «negli scritti del Vinci il tasto religioso, per quel che riguarda rivelazioni cristiane e ripensamenti di esse, è scrupolosamente evitato; se vi sono puntate ironiche o son contro la corrutela del clero, o in genere contro l'atteggiamento dogmatico mentale della filosofia e della scienza del tempo. Pare che ci tenesse molto, molto più che ad essere riservato in materia d'amore ${ }^{35}$. A sua volta Cesare Luporini osserva che negli scritti di Leonardo manca la teologia e dice del maestro toscano: «non che voglia con questo farne un ateo, ma certo un panteista e deista avanti lettera sì» ${ }^{36}$.

Delécluze affronta poi l'ultimo periodo della vita di Leonardo, nel quale egli nominò suo erede l'allievo Francesco Melzi ${ }^{37}$. Giunse quindi il momento della morte del pittore, che secondo la tradizione avvenne tra le braccia del re di Francia Francesco I. Alla base di questo episodio divenuto leggendario c'è Giorgio Vasari, che narrò nei seguenti termini il decesso di Leonardo:

«sopraggiunseli il re che spesso et amorevolmente lo soleva visitare; per il che egli per riverenza rizzatosi a sedere sul letto, contando il mal suo e gli accidenti di quello mostrava tuttavia quanto avea offeso Dio e gli uomini del mondo, non avendo operato come si conveniva. Onde gli venne un parossismo messaggero della morte. Per la qual cosa rizzatosi il re e presoli la testa per aiutarlo e porgeli favore, acciò che il male lo alleggerisse, lo spirito suo, che divinissimo era, conoscendo non potere avere maggiore onore, spirò in braccio a quel re nella età sua d'anni $75 \gg^{38}$.

(33) Ibidem. L'ingresso trionfale di Francesco I a Milano nel 1515 segnò la sua vittoria definitiva contro la Lega Santa, una coalizione antifrancese promossa nel 1511 dal Pontefice, alla quale aderirono la Spagna, l'Inghilterra e la Svizzera.

(34) Ibidem, p. 65. A proposito dei «défauts condamnables» delle «mœurs» di Leonardo, si potrebbe pensare, che Delécluze facesse riferimento all'omosessualità dell'artista. Tuttavia nel suo saggio l'autore non accenna al complesso problema della sessualità in Leonardo, che invece comincerà a destare interesse presso autori di sensibilità decadente, primo tra tutti Théophile Gautier.

(35) G. Fumagalli, Eros di Leonardo, Firenze, Sansoni, 1971, p. 203.
(36) C. Luporini, La mente di Leonardo, Firenze, Sansoni, 1953, p. 19.

(37) Le leggi francesi del tempo impedivano agli stranieri di trasmettere in eredità i beni che essi detenevano in Francia. Tuttavia Leonardo, avendo confidato al re il suo rammarico di non poter far testamento, ottenne dal sovrano stesso il permesso straordinario di disporre dei propri beni secondo la sua volontà. Francesco Melzi, in una lettera del $1^{\circ}$ giugno 1519, dichiarava che senza la benevolenza di Francesco I tutta l'eredità lasciata da Leonardo sarebbe andata perduta. (M.-T. MAIROT-DrOMARD, La vie de Léonard de Vinci au Clos-Lucé et l'bistoire de sa demeure, Imprimerie Saint-Paul, Bar le Duc, 1983 , p. 39).

(38) G. VASARI, op. cit., p. 407. 
Sulla scia del biografo italiano, molti studiosi francesi di Leonardo riportarono a loro volta l'episodio della sua morte tra le braccia di Francesco I. Anche Diderot, per esempio, narrò che: «Léonard de Vinci, peintre florentin, appellé à la cour de François $\mathrm{I}^{\mathrm{er}}$, ce prince le logea dans son château à Fontainebleau. Il l'aimait tant, que Léonard étant tombé malade, il allait le visiter souvent. Un jour comme le Roi entrait chez lui, Léonard de Vinci voulant se soulever pour lui témoigner sa reconnaissance, tomba en faiblesse; le Roi voulut le soutenir, et cet artiste expira dans ces bras» ${ }^{39}$. Il viaggiatore Lalande affermò addirittura che il re sorresse Leonardo per fargli bere del brodo e rimproverò un nobile che aveva espresso il suo stupore per il dolore manifestato dal sovrano a causa della scomparsa dell'artista: «François $\mathrm{I}^{\mathrm{er}}[\ldots]$ étoit venu le voir dans sa derniere maladie à Fontainebleau, en 1518, il mourut à l'instant que le Roi le soutenoit pour lui faire prendre un bouillon; le désespoir du Monarque étonnoit les courtisans; l'un d'eux osa marquer sa surprise, mais le Roi lui répondit avec indignation: je puis faire de Grands Seigneurs tous les jours, et Dieu seul peut faire l'homme que je perds» ${ }^{40}$. Tuttavia, all'inizio del XIX secolo, Jean-Baptiste Venturi, professore modenese di fisica trasferitosi a Parigi ed appassionatosi allo studio di Leonardo dopo averne visto i manoscritti depredati da Napoleone a Milano, condusse una serie di ricerche che smentirono l'antica leggenda. Le sue prove erano inconfutabili e Delécluze le riporta tutte: quando Leonardo morì la corte non si trovava ad Amboise ma a Saint-Germain-en-Laye, dove la regina aveva appena partorito; gli ordini emanati dal sovrano in quei giorni sono datati da quella località; i documenti di corte non parlano di alcun viaggio del sovrano fino al mese di luglio (mentre Leonardo morì il 2 maggio); le imminenti elezioni imperiali stavano impegnando molto Francesco I, dal momento che egli era uno dei candidati al trono; Melzi, inoltre, nella lettera in cui informa i fratelli di Leonardo della sua morte, non racconta che essa sia avvenuta in modo così onorevole. Nonostante l'inoppugnabilità degli argomenti addotti da Venturi, molti critici restarono fedeli alla leggenda secondo cui Leonardo morì tra le braccia di Francesco I. Delécluze riporta e accetta la verità storica, tuttavia, consapevole dell'esistenza di una radicata leggenda, commenta che «il y a des choses vraisemblables qui équivalent à la réalité. Léonard de Vinci était digne d'un tel honneur, et l'intérêt vif que François $\mathrm{I}^{\text {er }}$ a toujours montré pour les arts, les artistes et pour Léonard en particulier, est cause que l'erreur signalée par Venturi sera difficilement détruite ${ }^{41}$. Analogamente, nel 1835, un anonimo commentatore di un quadro di Gigoux raffigurante la morte di Leonardo tra le braccia del sovrano (fig. 2), aveva scritto che questa leggenda era «plus poétique et plus significative» della realtà, poiché «l'un des plus grands artistes de l'Italie mourant au milieu du plus beau palais de France, entre les bras du roi qui a su attacher son nom à la renaissance des arts, c'est là un sujet qui émeut et féconde la pensée, ne fût-ce que comme allégorie» ${ }^{42}$.

Il saggio di Delécluze prosegue con alcune pagine dedicate agli scritti di Leonardo da Vinci. L'autore afferma che «on conserve treize manuscrits, dont l'un, qui est à

(39) D. Diderot, Salons, a cura di J. Seznec e J. Adhémar, Oxford, Clarendon Press, 1957-1967, vol. IV, p. 369. Diderot compie un errore piuttosto frequente nelle biografie leonardesche del Settecento, cioè afferma che l'artista morì a Fontainebleau. In realtà ai tempi di Leonardo questa residenza non era che un modesto castello, al quale Francesco I cominciò ad interessarsi solo a partire dal 1528 e iniziò a chiamare artisti italiani per decorarlo solo dal 1530. E possibile che a suggerire l'errore di Diderot e di altri biografi (Lacombe,
Dézallier d'Argenville, Lalande) sia stato Vasari, il quale dichiarava che la Gioconda era conservata a Fontainebleau.

(40) J.-J. DE LaLande, Voyage d'un françois en Italie, fait dans les années 1765 et 1766 , Yverdon, 1769 , p. 258. Per quanto riguarda Fontainebleau si veda la nota precedente.

(41) É.-J. DelÉCLUZE, op. cit., p. 67.

(42) «Magasin Pittoresque», troisième année, Paris, Imprimerie de Bourgogne et Martinet, 1835, p. 78 . 


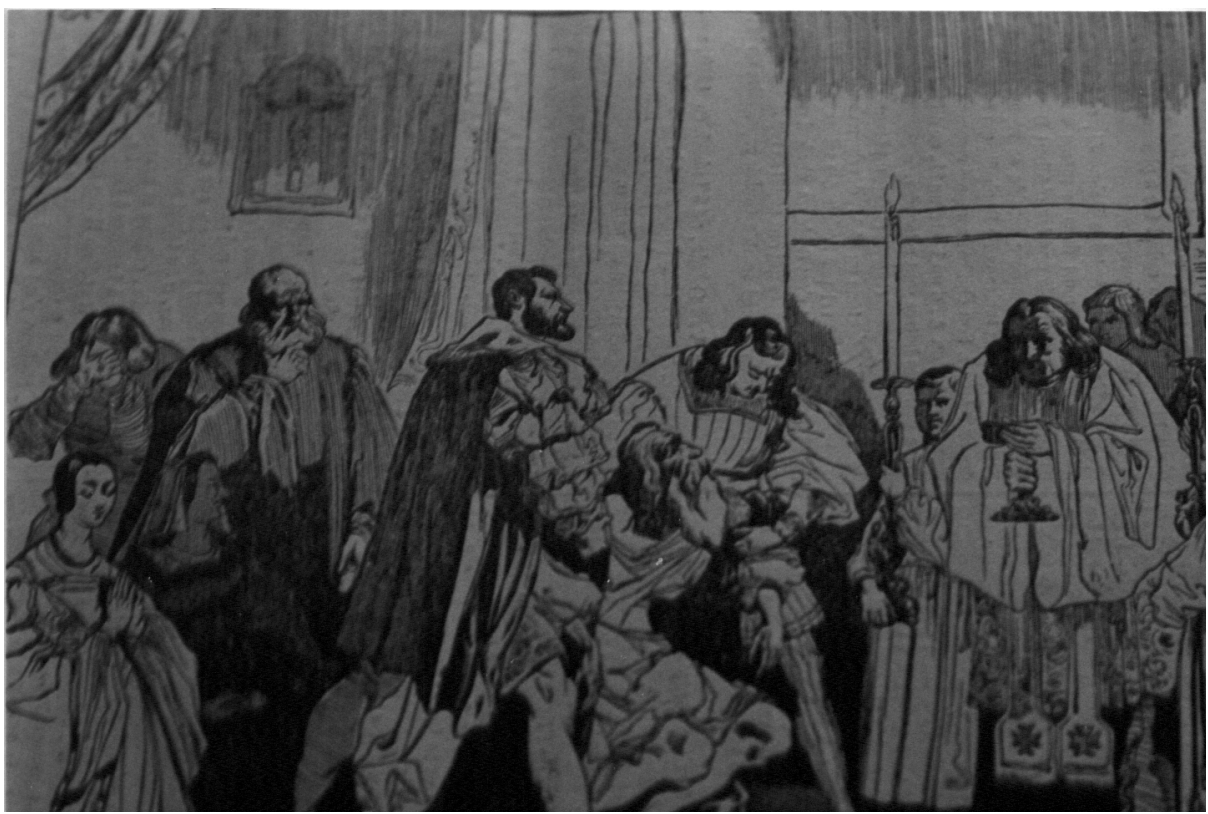

la Bibliothèque Ambroisienne, à Milan, est de format atlantique et de l'épaisseur de quatre ou cinq pouces. Tous écrits à la manière des Orientaux, de droite à gauche, ces manuscrits se composent de feuillets sur lesquels Léonard [...] avait coutume de faire des notes, des croquis, des démonstrations scientifiques» ${ }^{43}$. I tredici manoscritti di cui parla Delécluze sono quelli che Napoleone depredò a Milano e portò a Parigi nel 1796, dei quali il Codice Atlantico tornò a Milano dopo la Restaurazione. È curioso constatare che l'osservazione secondo cui Leonardo scriveva «à la manière des Orientaux», cioè da destra a sinistra, non è nuova. Infatti, proprio al momento della restituzione del Codice Atlantico all'Italia nel 1815, il commissario austriaco Ottenfels «fu sul punto di lasciare alla Biblioteca Nazionale di Parigi il Codice Atlantico perché, osservando la strana grafia inversa di Leonardo, l'aveva scambiato per un testo cinese» ${ }^{44}$. Fortunatamente Antonio Canova e i commissari del Granduca di Toscana lo costrinsero a ritirare il Codice. Anche il viaggiatore ottocentesco Valéry, che vide il Codice Atlantico nella sua sede milanese nel 1826, aveva scritto che in esso «les lettres sont tracées de droite à gauche, à la manière des Orientaux, et ne peuvent êtres lues que au miroir» ${ }^{45}$. Delécluze mette in evidenza che di tutti i preziosi frammenti dei trattati leonardeschi giunti fino a noi, solamente quello sulla pittura è stato pubblicato. Tuttavia a suo avviso il titolo Traité de peinture non è appropriato per quest'opera, in quanto si tratta in realtà di una «compilation, faite sans ordre, des pensées et des observations qui se rapportent bien en effet au traité complet de peinture, tel que Léonard l'avait conçu, mais qui isolées et inverties, forment un assemblage indigeste et confus de remarques ou de règles [...] qui ne se coordonnent

(43) É.-J. DeléCluZe, op. cit., p. 67.

(44) P.C. Marani, M. Rossi, A. Rovetta, L'Ambrosiana e Leonardo, Milano, Veneranda Biblioteca Ambrosiana, 1998, p. 13.
(45) VALERY, Voyages historiques et littéraires en Italie, pendant les années 1826, 1827, 1828, ou l'Indicateur Italien, Paris, Le Normand Librairie, 1831, p. 148. 
pas entre elles» ${ }^{46}$. Il critico ottocentesco, però, prosegue affermando che nonostante il suo carattere asistematico, il Traité de peinture di Leonardo è il testo tuttora più utile per la formazione dei giovani pittori. Nelle pagine seguenti Delécluze riporta alcuni passaggi tratti dai frammenti delle opere leonardesche, che egli ha conosciuto grazie al lavoro di Venturi. I brani che l'autore sceglie di trascrivere nel suo saggio (che qui vengono tralasciati) mirano a far risaltare la figura di Leonardo come scienziato che segue una metodologia rigorosa, non lascia nulla al caso, sottoponendo all'esperienza e all'osservazione ogni sua ipotesi. Secondo Delécluze, Leonardo sarebbe stato un grande geologo e il precursore di alcune importanti invenzioni, quali il barometro, la camera oscura e le macchine a vapore. Egli mette in evidenza che il principale obiettivo di Leonardo consisteva nel riuscire a soddisfare al bisogno di «augmenter et accélerer les forces de l'homme» ${ }^{47}$.

Giunto alla fine della sua biografia leonardesca, Delécluze ritorna alla metafora da cui era partito, cioè quella che accostava l'arte ai fiori. All'inizio dello scritto era emerso che i fiori sono uno stadio intermedio, il cui prodotto finale è la pianta, mentre non era stato indicato il fine ultimo delle arti. Ora Delécluze lo svela, partendo da una riflessione su Leonardo:

«quant à Léonard de Vinci, je dois dire que c'était avant tout un savant, mais que, doué tout à la fois d'un admirable instinct d'artiste, il semble s'être servi de cette seconde faculté pour essayer d'abord, puis affermir ensuite la première. C'est avec amour qu'il a cultivé la fleur, mais non sans la prévoyance constante qu'elle devait produire un fruit. Or ce fruit, qui n'est autre chose que la science, fut pendant la vie entière de Léonard l'objet de tous ses soins, de toutes ses espérances» ${ }^{48}$.

Il saggio di Delécluze costituisce una tappa importante nella storia della critica di Leonardo in Francia, poiché con questo scrittore comincia a farsi strada un nuovo modo di concepire il grande artista toscano. Gli autori francesi del Settecento, tra cui Jaucourt, Watelet e Lévesque, infatti, avevano dato di lui un'immagine statica e quasi stereotipa: quella del genio che ha ricevuto dalla natura talenti superiori e li ha fatti fruttare soprattutto in campo scientifico. Altri biografi, come Lacombe, Dézallier d'Argenville e Mariette, si erano limitati a ripercorrere la vita del maestro elencando le sue opere più importanti e riportando aneddoti leggendari separati l'uno dall'altro. All'inizio dell'Ottocento, invece, Gault de Saint-Germain aveva scritto una bella biografia di Leonardo, ma a sua volta aveva dato del maestro un'immagine non realistica, quella che corrisponde al topos letterario dell'intellettuale perseguitato, del genio destinato ad affrontare l'invidia e l'ostilità del mondo perché la sua infelicità terrena è il prezzo che egli deve pagare per i privilegi che la natura gli ha concesso. Nel saggio di Delécluze, invece, la vita di Leonardo viene analizzata in modo il più possibile attinente alla verità. In questo scritto l'artista è sempre presentato come un grande genio, ma gli episodi della sua vita vengono ora inseriti in un contesto organico e soprattutto sono motivati da considerazioni di carattere storico. Leggendo queste pagine si ha

(46) É.-J. Delécluze, op. cit., p. 67. L'opera di Leonardo conosciuta come Trattato di Pittura aveva in realtà per titolo Libro di Pittura, ma nella seconda metà del Cinquecento qualcuno abbreviò il testo leonardesco riducendo i suoi 944 precetti a soli 365 e dando a questo manuale la denominazione di Trattato. Carlo Perdetti afferma che «se è vero che il termine trattato è del tutto estraneo ai propositi che informavano l'attività teorica di Leonardo-per il quale il libro è sempre e prima di tutto uno strumento di registrazione-, è anche vero che bene risponde alle esigenze didattiche delle botteghe alle quali compete il ruolo di scuola e che si adeguano quindi alla funzione svolta più tardi dalle Accademie» (LeONARDO DA VINCI, Libro di pittura, Codice Urbinate latino 1270 nella Biblioteca apostolica Vaticana, a cura di Carlo Perdetti e Carlo Vecce, Firenze, Giunti, 1995, vol. I, p. 43-44).

(47) É.-J. DelÉCluze, op. cit., p. 76.

(48) Ibidem, p. 79. 
l'impressione di entrare in contatto con un Leonardo un po' meno leggendario, forse meno affascinante, ma sicuramente più umano di quello presentato dalla critica precedente a Delécluze. Come si è già avuto modo di evidenziare, inoltre, l'autore fu sempre ostile agli ideali del romanticismo. Nelle sua teoria dell'arte come strumento per arrivare alla scienza si può ravvisare un segno della concretezza del suo pensiero e un germe del positivismo che si affermerà nella seconda metà del secolo. In quest'ottica le arti figurative non sono fini a loro stesse, ma hanno un obiettivo ultimo che consiste nel contribuire al progresso umano identificato nella scienza. Ecco perché l'autore ha scelto proprio Leonardo come oggetto del suo approfondimento, scrivendone una biografia corretta, vasta e vagliata criticamente. I suoi giudizi in merito all'arte del pittore sono scarsi e in linea di massima sono conformi al gusto del tempo, che individuava nel «Cenacolo» l'opera leonardesca più pregevole. Anche il paragone con gli altri grandi artisti del tempo, Michelangelo e Raffaello in prima istanza, costituisce un topos della critica d'arte del Sette-Ottocento relativa al grande maestro toscano, tuttavia Delécluze lo ha trattato con risvolti nuovi e originali, integrandolo nel suo studio del contesto storico in cui visse Leonardo.

WILMA PROGLIO 